\title{
FINAL MEASUREMENTS OF THE SRS MULTIPOLE WIGGLERS
}

\author{
J.A. Clarke, N. Bliss, D.J. Bradshaw, N.W. Harris, C.L. Hodgkinson, R. Marl, I.D. Mullacrane, \\ M.W. Poole, M.J. Pugh and R.J. Reid, CLRC Daresbury Laboratory, Warrington, UK WA4 4AD.
}

\begin{abstract}
Two new high field $2 \mathrm{~T}$ multipole wigglers have been built for the SRS. Detailed mechanical and magnetic tests have been made and these are compared with the expected theoretical performance. These insertion devices have now been installed into the SRS and are operating successfully.
\end{abstract}

\section{INTRODUCTION}

Two new high field (2 T) hybrid Multipole Wigglers have been designed and constructed for the SRS Upgrade Project [1]. The two insertion devices are identical and have been specified to provide maximum flux at a photon energy of $10 \mathrm{keV}$. The chosen design will provide a source with about 25 times more photons per horizontal angle at this photon energy than a standard SRS dipole source. One of the beamlines will have 2 experimental stations, both dedicated to protein crystallography. The other beamline will initially have one station, for studying the interaction of photons with molecules, nanoclusters and surfaces, although provision has been made for a $2^{\text {nd }}$ station to be added at a later date.

All aspects of the Multipole Wigglers were fully specified and designed by Daresbury Laboratory but the manufacture and assembly was carried out under a commercial contract by Sincrotrone Trieste SCpA. The contract was placed during the Summer of 1997 and both the insertion devices were delivered to Daresbury Laboratory 11 months later. The final magnet design is described in [2].

\section{MECHANICAL MEASUREMENTS}

A detailed series of mechanical measurements were carried out on both MPWs (referred to as MPW 1 and MPW 2) after they were delivered to Daresbury Laboratory. The gap between the steel pole pieces was set to the nominal minimum value of $19.2 \mathrm{~mm}$ and ceramic slip gauges with increments of $1 \mu \mathrm{m}$ were used to measure the gap under each of the 11 poles in two horizontal positions. As expected the gap was slightly larger on the inside of the magnet compared to the outside. This is simply due to the $\mathrm{C}$ shape design of the magnet support structure. The maximum gap difference along the whole of the magnet was $40 \mu \mathrm{m}$ for MPW 1 and $102 \mu \mathrm{m}$ for MPW 2. This compares remarkably well with the predicted $80 \mu \mathrm{m}$ from FEA calculations [3].
Measurements were also made of the repeatability of the carriages between the maximum gap of $200 \mathrm{~mm}$ and 19.2 $\mathrm{mm}$. The pitch and roll were measured with a Kern Nivel 20 electronic level with a resolution of $1 \mu \mathrm{rad}$. The gap was opened and closed 5 times and the maximum angle variation observed at either gap for MPW 1 was $6 \mu \mathrm{rad}$ of pitch and $11 \mu \mathrm{rad}$ of roll and for MPW 2, $11 \mu \mathrm{rad}$ of pitch and $8 \mu \mathrm{rad}$ of roll. In each case the repeatability is excellent for a mechanical system where the force between the two arrays is increasing by about 5 tonnes as the gap closes.

\section{MAGNETIC MEASUREMENT LABORATORY}

A new Magnet Measurement Laboratory has been built at Daresbury Laboratory for measuring insertion devices. Two measurement facilities were designed, a Hall Probe Bench and a Flipping Coil Bench. The laboratory is temperature controlled to $\pm 1^{\circ} \mathrm{C}$ to improve the repeatability of the measurements.

\subsection{Hall Probe Bench}

Table 1. Hall Probe Bench specifications.

\begin{tabular}{|l|l|}
\hline & MPT-141-3m \\
Hall Probe & DTM-141-DG \\
Leslameter & $1400 \mathrm{~mm}$ \\
Horizontal Range & $200 \mathrm{~mm}$ \\
Vertical Range & $100 \mathrm{~mm}$ \\
& \\
Longitudinal Resolution & $1 \mu \mathrm{m}$ \\
Horizontal Resolution & $0.5 \mu \mathrm{m}$ \\
Vertical Resolution & $0.5 \mu \mathrm{m}$ \\
& \\
Nominal Longitudinal Velocity & $1 \mathrm{~mm} / \mathrm{s}$ \\
& \\
Maximum Calibrated Field & $2.2 \mathrm{~T}$ \\
Hall Probe Precision & $\pm 0.01 \%$ \\
Hall Probe Resolution & $0.5 \mathrm{G}$ \\
Temperature Stability & $\pm 10 \mathrm{ppm} /{ }^{\circ} \mathrm{C}$ \\
Sensitive Area & $1.0 \mathrm{x} 0.5 \mathrm{~mm}$ \\
& \\
\hline
\end{tabular}

The Hall Probe Bench is designed to measure the magnetic field at discrete points in the insertion device. The Hall Probe can be scanned in all three linear axes with a resolution of $1 \mu \mathrm{m}$ in the longitudinal direction and 
$0.5 \mu \mathrm{m}$ in the horizontal and vertical directions. The Hall Probe and Teslameter used were supplied by Group 3 [4]. The specification of the Hall Probe System is summarised in Table 1. The system is fully automated and is controlled by a local PC running LabVIEW [5]. The PC uses RS232 to interface with the motors and encoders and GPIB to interface with the Hall Probe. The Hall Probe is normally oriented to measure vertical magnetic fields though it can measure in other orientations as required. At present the system moves the probe to the desired location and then takes a reading once it has stopped moving. The possibility of an 'on-the-fly' measurement has not yet been investigated. The time taken for a $1.4 \mathrm{~m}$ scan with a reading every $2 \mathrm{~mm}$ is approximately 30 minutes (less than $3 \mathrm{~s}$ per point).

\subsection{Flipping Coil Bench}

The Hall Probe system is designed to measure the field at discrete points, so it is ideal for mapping out the magnetic field along the axis, for example. However, since each field reading has an error associated with it (albeit small) the total error in any integral measurement can be significant. In order to measure the field integrals more accurately a flipping coil scheme was also designed. This will measure the integrals in a single measurement (in a few seconds) and the error in the measurement should be smaller than in a numerically calculated integral taken from the Hall Probe scan. A 20 turn coil, made by soldering individual $50 \mu \mathrm{m}$ strands of Litz wire together to form a complete circuit, is mounted between two rotating heads. The coil mounting heads are approximately $2.5 \mathrm{~m}$ apart on a linear guide rail system. They can be moved manually in a longitudinal direction so that the correct tension can be set in the wire; a tensiometer is provided for this purpose. The heads can be moved remotely in the horizontal and vertical directions with a resolution of 0.5 $\mu \mathrm{m}$. The heads can also rotate $360^{\circ}$ about the longitudinal axis with a resolution of better than $0.001^{\circ}$. The full specification for the flipping coil system is given in Table 2. The system is fully automated and is controlled by a local PC running LabVIEW. The PC uses RS232 to interface with the motors and encoders and GPIB to interface with the HP Multimeter that is used as the Voltage Integrator.

The flipping coil system was only completed as the MPWs arrived for testing, therefore the commissioning time available was very short. The system gave reasonable results but the repeatability was never better than a few \%. It became apparent that the repeatability was limited because of a noisy voltage signal from the coil. The noise appeared to be due to the coil vibrating as it was rotated. Vibration measurements have recently started to try to determine exactly which component of the system is responsible for the vibration so that remedial action can be taken. The integrated field measurements presented in this paper were all calculated from the Hall Probe results.

Table 2. Specifications for the Flipping Coil Bench

\begin{tabular}{|l|l|}
\hline & \\
Nominal Coil Length & $2.5 \mathrm{~m}$ \\
Maximum Coil Length & $3.0 \mathrm{~m}$ \\
Nominal Coil Width & $10 \mathrm{~mm}$ \\
Nominal Rotation Velocity & $20 \% \mathrm{~s}$ \\
& \\
Horizontal Range & $200 \mathrm{~mm}$ \\
Vertical Range & $100 \mathrm{~mm}$ \\
& \\
Rotation Resolution & $<0.001^{\circ}$ \\
Horizontal Resolution & $0.5 \mu \mathrm{m}$ \\
Vertical Resolution & $0.5 \mu \mathrm{m}$ \\
Voltage Integrator & HP 3458A DMM \\
\hline
\end{tabular}

\section{MAGNET MEASUREMENT RESULTS}

The peak field measured under the central pole as a function of gap is given in figure 1. The maximum field variation between the 9 full strength poles at the minimum gap of $19.2 \mathrm{~mm}$ is less than $0.5 \%$ for both magnets.

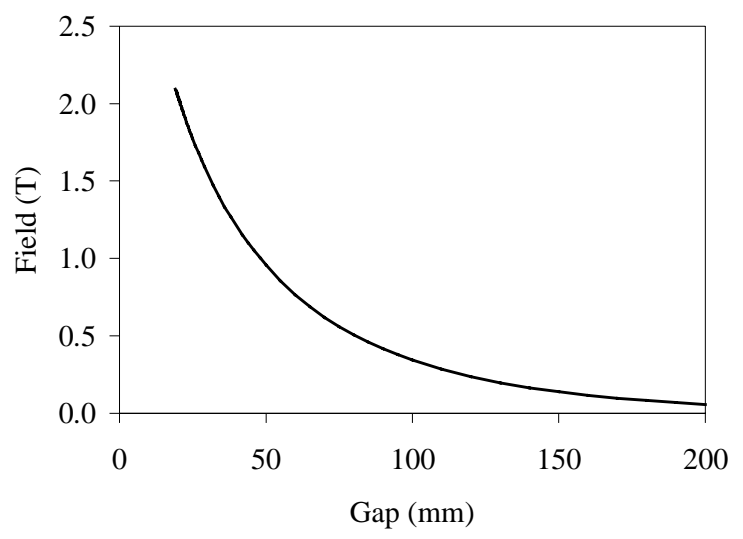

Figure 1. Measured peak field on axis against gap.

The vertical magnetic field as a function of longitudinal position at $19.2 \mathrm{~mm}$ gap is shown in figure 2. The full strength poles reach a field of $2.09 \mathrm{~T}$ and the two end poles are $1.71 \mathrm{~T}$. The agreement with the threedimensional model generated with Opera 3D [6] is very good with the largest difference between the two sets of data at the poles being $0.9 \%$. The measured and modelled data for the central portion of one of the MPWs is shown in figure 3 . 


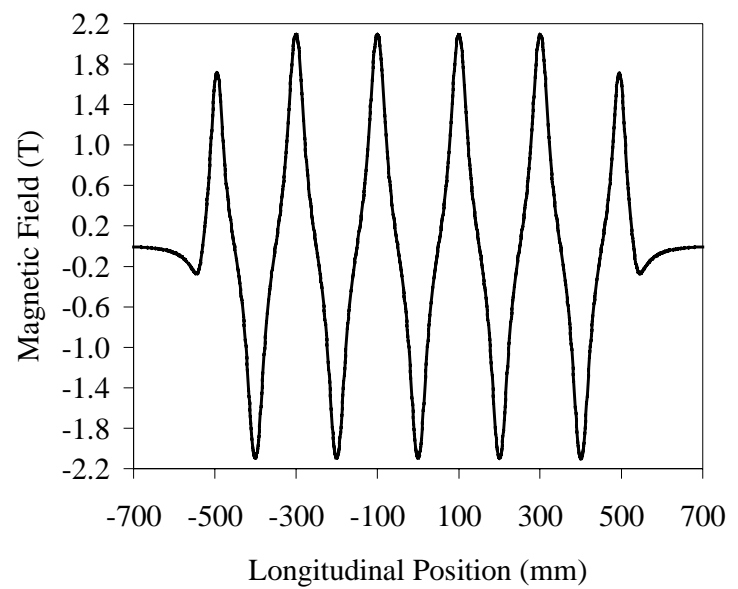

Figure 2. Measured field on axis against longitudinal position at $19.2 \mathrm{~mm}$ gap.

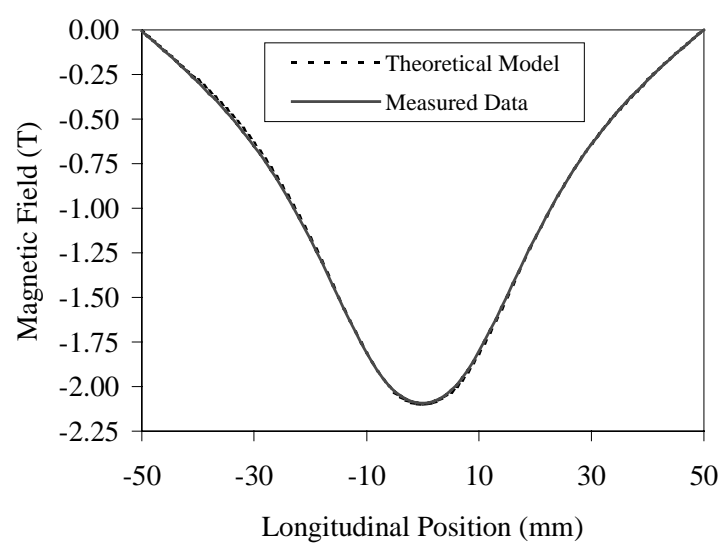

Figure 3. Comparison of the measured field values with the theoretical model over the central pole at $19.2 \mathrm{~mm}$ gap.

The measured field integrated longitudinally at different horizontal offsets is given in figure 4 for both MPWs. A $6^{\text {th }}$ order polynomial has been fitted to the data points to estimate the integrated sextupole. For MPW 1 the integrated sextupole at minimum gap is $1.37 \mathrm{~T} / \mathrm{m}$ and for MPW 2 it is $2.60 \mathrm{~T} / \mathrm{m}$. The difference between the two magnets is due to the greater angle error between the two magnet arrays in the radial direction in MPW 2 compared with MPW 1 as discussed in Section 2.

The variation of the integrated vertical field on axis as a function of gap is plotted in figure 5. The maximum integral of $2.0 \mathrm{mT}-\mathrm{m}$ is easily compensated with the simple trim coil fitted to the MPWs. The maximum current required in the trim coils to maintain a no steering condition from the MPW is about $0.5 \mathrm{~A}$, giving a current density within the air-cooled copper conductor of about $0.2 \mathrm{~A} / \mathrm{mm}^{2}$.

Both MPWs are now installed in the SRS and commissioning with beam has commenced [7]. Since the MPWs exceed the specified requirement of the beamlines to operate at $2.0 \mathrm{~T}$ the operating gap will be $20.5 \mathrm{~mm}$ instead of the minimum of $19.2 \mathrm{~mm}$.

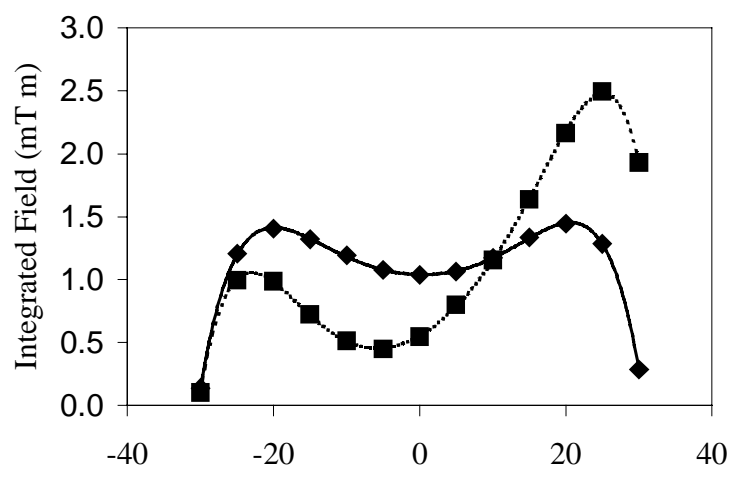

Horizontal Position (mm)

Figure 4. Integrated vertical field against horizontal position with a $6^{\text {th }}$ order polynomial fit. The solid line is for MPW 1 and the dashed line is MPW 2.

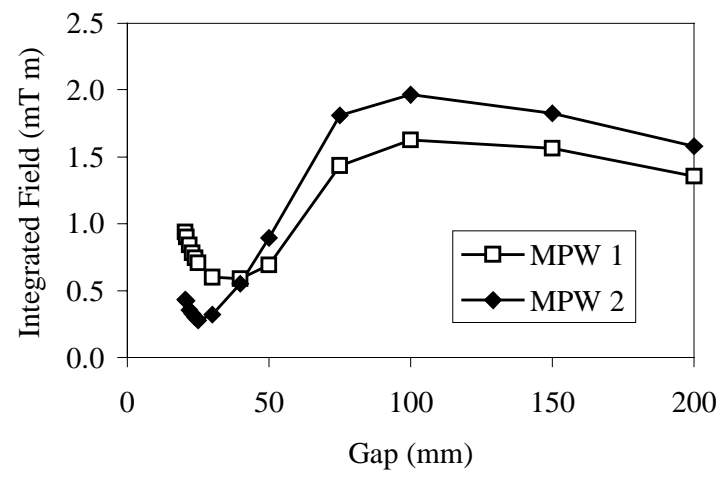

Figure 5. Integrated vertical field on axis as a function of gap.

\section{REFERENCES}

[1] J.A. Clarke and M.W. Poole, "Upgrading the Daresbury SRS with Additional Insertion Devices and its Implications for the Storage Ring Layout", Proc. $5^{\text {th }}$ Euro. Part. Accel. Conf., Sitges, June 1996, p623.

[2] J.A. Clarke et al, "Progress with the SRS Upgrade Project", Proc. $6^{\text {th }}$ Euro. Part. Accel. Conf., Stockholm, June 1998, p571.

[3] J.A. Clarke et al, "Design of a $2 \mathrm{~T}$ Multipole Wiggler Insertion Device for the SRS”, J. Synchrotron Rad. (1998). 5, p434.

[4] Group 3 Technology Ltd, Rosebank, Auckland, New Zealand.

[5] National Instruments, Austin, Texas, USA.

[6] Vector Fields, Kidlington, Oxford, UK.

[7] J.A. Clarke and G.S. Dobbing, "Commissioning of the New Multipole Wigglers in the SRS", these proceedings. 